accepted for publication in Health Psychology

cite as: de Visser, R.O., Graber, R., Hart, A., Abraham, C., Memon, A., Watten, P. \& Scanlon, T. (in press) Using qualitative methods within a mixed-methods approach to developing and evaluating interventions to address harmful alcohol use among young people. Health Psychology.

note: final published version may vary

\title{
Using Qualitative Methods within a Mixed-Methods Approach to Developing and Evaluating Interventions to Address Harmful Alcohol Use among Young People
}

Objective: This paper illustrates how qualitative methods can be used in the development and evaluation of behavior change interventions. Although many campaigns advise young people to drink responsibly, few clarify how to convert this general advice into specific behavioral strategies. Resilience-based approaches argue that treating young non-drinkers and moderate drinkers as "experts" in responsible alcohol use may facilitate co-creation of acceptable interventions that focus on how to change behavior. Methods: Four distinct phases of intervention development were linked to past research and future developments. Results: First, analysis of correlates of alcohol use using data from a survey of 1412 people aged 16-21 indicated that alcohol harm-reduction interventions should help young people to develop skills and strategies to resist alcohol. Second, Interpretative Phenomenological Analysis of 25 interviews with people purposively-selected from among the survey sample identified general strategies and specific tactics used by young people to manage opportunities to drink. Third, insights from the first two phases and past qualitative research guided development of video resources to be use in school-based alcohol education to illustrate strategies and tactics for moderate- or non-use of alcohol. Fourth, 18 focus groups with students and teachers were used to evaluate the video: structured Thematic Analysis indicated that after revision the video would be a valuable addition to school-based alcohol education. Conclusions: Findings from the four phases highlight the value of using different qualitative and quantitative methods as part of a program of work designed to inform the development, refinement, and evaluation of health psychology interventions.

Keywords: intervention; qualitative; video; school; alcohol; peer education 
Qualitative methods that focus on lived experience may offer invaluable insight into young people's experiences of non-drinking and moderate drinking in a "binge drinking” youth culture (e.g., Conroy \& de Visser, in press; Frederickson, Bakke \& Dalum, 2012; Robertson et al., 2012). Strategic use of multiple qualitative methods may aid the development and evaluation of interventions that support young people to achieve and maintain moderate levels of alcohol consumption, or to abstain from alcohol. The study reported here provides an illustrative case study.

In recent decades, principles of social marketing have been applied with success in health psychology and public health (Gordon, McDermott, Stead \& Angus, 2006). Unlike massmedia campaigns concerned solely with information provision/control, social marketing involves an integrated series of activities employed in an iterative and responsive manner to ensure that the message and the mode of delivery are meaningful and appealing (Cheng, Kotler \& Lee, 2011; Gordon et al., 2006; Randolph \& Viswanath, 2004). Key elements of social marketing include: population segmentation to identify a specific target group; use of quantitative and/or qualitative methods to understand people's values and needs and to inform intervention development; an understanding of the principle of exchange and demonstration of appealing pay-offs from behaviour change; analysis of appropriate communication channels; formative evaluation of the intervention by the target audience during its development; and implementation and formal evaluation of the final revised intervention

A systematic review concluded that social marketing interventions can reduce alcohol misuse, and included evidence that school-based social marketing can influence alcohol use among young people (Gordon et al., 2006). Furthermore, a recent systematic review indicated that videos can be an effective component of interventions, especially if they: are tailored to the target audience; use gain-framed messages that emphasize the benefits of changing behavior; and include modeling of desired behavior (Tuong, Larsen \& Armstrong, 2014). That systematic review identified no studies that had included videos as a key component of interventions designed to address alcohol use among young people (Tuong et al., 2014).

The aim of this paper is to illustrate how different modes of qualitative research can be integrated, and combined with quantitative methods in a work program designed to inform the development and revision of effective health psychology interventions.

Young people and alcohol

There is widespread concern about the health and social consequences of excessive alcohol consumption among young people - i.e., those aged 15-21 years (Babor et al., 2010). Much attention has been given to heavy episodic drinking (HED), also known as "binge drinking" 
(Herring, Berridge \& Thom, 2008), which is associated with an increased risk of alcohol poisoning, accidents and injury, and being the victim or perpetrator of violence (Boles \& Miotto, 2003; Cherpitel et al., 2003). Across developed countries, young people are the population segment most likely to report HED (White et al., 2011). However HED is not the only marker of problematic drinking, and it is important to consider subject definitions of drinking "too much".

Many existing health promotion campaigns encourage young people to drink responsibly, but do not clarify how to convert this general advice into specific behavioral strategies or offer realistic models of behavior change (Gordon et al., 2006). One notable exception to this in the UK is the National Health Service (NHS) "Change4Life" campaign, which offers practical advice to individuals wishing to reduce their alcohol intake (NHS, 2012). The lack of attention to helping young people to identify and prepare for situations that may lead to heavy drinking is odd given that such skills are an important part of relapse prevention (Hendershot, Witkiewitz, George \& Marlatt, 2011; Larimer, Palmer \& Marlatt, 1999).

In recent qualitative research with young people, public health initiatives have been criticized for focusing too much on physical risks and harms to health - which young people tend not to worry about - and not giving sufficient attention to the pleasure arising from drinking - which is important to young drinkers (de Visser, Wheeler, Abraham \& Smith, 2013; Fry, 2011; Harrison, Kelly, Lindsay, Advocat, \& Hickey, 2011; Szmigin et al., 2008).

Conventional campaigns also tend to focus on individual responsibility and ignore the group-based social nature of drinking (de Visser et al., 2013; Fry, 2011; Harrison et al., 2011; Hutton, 2012; Jayne et al., 2010; Szmigin et al., 2011). Therefore, campaigns that target individual harms arising from HED may be dismissed as irrelevant, particularly given that many are perceived to employ a patronizing, paternalistic, or preaching tone (de Visser et al., 2013). Peer group drinking and drunkenness are important aspects of the social lives of many young people: the individual experience of intoxication is often accompanied by enhanced feelings of togetherness (Brown \& Gregg, 2012; de Visser et al., 2013; Fry, 2011; Jayne et al., 2010; Livingstone, Young \& Manstead, 2011; MacNeela \& Bredin, 2011; Szmigin et al., 2011; Thurnell-Read, 2011). Furthermore, recent qualitative research has revealed that many young people believe that some negative aspects of drinking - such as caring for drunk friends, being cared for when drunk, and suffering through hangovers with friends - offer opportunities for strengthening interpersonal bonds (de Visser et al., 2013). Public health interventions should, therefore, address these important social motives.

Behavioral Skills and Resilience 
Effective alcohol education is vital for ensuring that young people are equipped with the information and skills needed to enact informed healthy choices about alcohol (Department for Education, 2011). The National Institute for Health \& Care Excellence (NICE: 2007) recommends "safer drinking" lessons for teenagers, based on evidence that such approaches are cost-effective (McKay et al., 2012). However, there is a lack of tested resources that teach or illustrate strategies or techniques to use to resist alcohol.

The Information-Motivation-Behavioral Skills model (Fisher, Fisher, Williams \& Malloy, 1994) highlights the need not only to improve knowledge of the harms related to excessive alcohol use, but to develop skills to enable this knowledge to be put into practice. Possession of such skills is reflected in drink-refusal self-efficacy (DRSE), which is linked to reduced risk of harmful drinking (Atwell et al., 2011; Baldwin et al., 1993).

A psychosocial resilience framework argues that it is important to develop skills, selfconfidence, and protective mechanisms to help people to manage challenging situations and adversity (Hart et al., 2007). When applied to the current behavioral focus, the challenging context is a youth "binge-drinking" culture, the required skills and self-confidence are conceptualized as DRSE, and the broader protective mechanisms include support for non- or moderate-drinking. (Johnson et al., 1998). A resilience approach moves beyond conceptualizing alcohol management skills solely as avoiding risky behaviors, and toward identifying protective mechanisms that support healthier patterns of alcohol use (Rutter, 1990). The resilience philosophy underlying the research reported here argues that treating young non-drinkers and moderate drinkers as "experts" in responsible drinking may facilitate co-creation of acceptable and feasible interventions that focus on how to change behavior. This involves learning how young people successfully manage responsible alcohol use in varied social situations, and using what is learnt to develop tailored interventions.

\section{Peer Influence and Peers as Educators}

Treating young people as a valuable resource (rather than simply as a problem group) is central to peer education. In developing health promotion materials for alcohol use, it is important to incorporate peer influences, because perceived peer behavior influences young people's alcohol use (Borsari \& Carey, 2003; França et al., 2010). Furthermore, young people who hold more negative perceptions of non-drinkers tend to report heavier alcohol consumption (Regan \& Morrison, 2013). It is therefore important to present positive images of moderate- and non-drinkers.

Peer educators can be credible "opinion leaders" who enact behaviors that their peers would seek to emulate (Kelly et al., 1992). This approach has been applied with some success 
in school-based peer-education interventions for health behaviors (e.g., Starkey et al., 2009). Peer-delivered health education may be perceived as more appealing than teacher-delivered health education (Stephenson et al., 2004), and may address young people's views that alcohol education tends to be patronizing or paternalistic (de Visser et al., 2013)

Peer education can also provide opportunities for young people to develop a critical consciousness of how socially constructed norms place their health at risk, and encourage the development of alternative norms (Campbell \& MacPhail, 2002). This perspective can be aligned with a resilience framework that highlights the importance of developing not only individual capacities, but also broader protective mechanisms including micro- and macrosocial support for non- or moderate-drinking. For example, the school environment can be an important influence on student health (Bonell et al., 2013).

The value of qualitative methods for developing and evaluation interventions

As indicated above, qualitative methods should be a key component of interventions developed according to social marketing principles (Cheng et al., 2011; Gordon et al., 2006). They can also provide information that is useful for the evaluation of the efficacy and acceptability of interventions, to determine whether proposed new interventions are perceived by young people and health educators to be useful, feasible, and credible, and can be used to elicit feedback to guide the revision of intervention materials (Lewin, Glenton \& Oxman, 2009; MacPherson \& McKie, 2010). Qualitative methods can also be combined with quantitative methods in process evaluation in trials of new interventions to examine the views of people who deliver and/or receive the intervention, and to investigate contextual factors that may affect intervention implementation and intervention efficacy in different sites (Oakley et al., 2006). Qualitative process evaluation can help to determine why effective interventions work, and whether less successful interventions are inherently flawed in conceptual terms, or are well-conceived but badly delivered.

This paper

The aim of this paper is to provide an illustration of how multiple qualitative methods can be combined and used within a resilience framework to develop and evaluate strengths-based behavior change interventions to prevent harmful alcohol use among young people. The research involved four distinct phases within a continuum linking insights from past qualitative research described above to future developments (Figure 1). The program of new work described here entailed the rigorous application of different qualitative methods in different phases and for different research purposes. The sections below describe a program of research designed to reduce alcohol consumption and alcohol-related harm among young 
people by enhancing DRSE and psychosocial resilience in a heavy-drinking culture. The focus was not limited to HED, but included subject definitions of drinking "too much". The goal was to produce materials for use in schools and to determine their appropriateness for the target audience of 15-17 years olds. The principles and processes described here could be applied to other health behaviors that are also an important part of young people's social lives such as smoking, and sexual behavior (Cheng et al, 2011).

\section{$>$ Figure $1<$}

\section{Phase 1}

A cross-sectional survey was conducted to identify patterns of alcohol consumption and their correlates among young people. Findings informed subsequent phases by: determining whether enhancing DRSE was a useful focus for the intervention; informing the development of the topic guide for Phase 2 interviews, and enabling purposive sampling of interviewees.

\section{$\underline{\text { Methods }}$}

An online questionnaire was completed by 776 women and 642 men aged 16-21 living in South-East England. Full details of the methods and sample demographics are reported elsewhere (Author, 2014a). Links to the survey homepage were distributed through: emails containing a link to the survey distributed in two universities; advertisements on the websites of 17 secondary schools; and distribution of promotional cards at 4 young people's services.

Respondents indicated whether they had ever consumed alcohol. Those who had then indicated whether they had consumed alcohol in the last year. Those who had then completed the 10-item Alcohol Use Disorders Identification Test (Babor, Higgins-Biddle, Saunders \& Monteiro, 2001). AUDIT scores and the two alcohol use history questions were used to allocate respondents to one of four alcohol use groups: non-drinkers (5\%), former-drinkers (5\%), low-risk drinkers (46\%), and hazardous drinkers (44\%).

Participants used 7-point scales ("strongly disagree" - "strongly disagree") to respond to the statement "Concerns about my health exert a strong influence over my use of alcohol" (de Visser \& McDonnell, 2012).

DRSE was assessed with 16 items (Young et al., 1991). Respondents used 7-point scales ("very difficult" - "very easy") to indicate their ability to refuse alcohol in 16 scenarios (e.g., "When someone offers me a drink"), with higher scores indicating greater DRSE ( $\alpha=.94)$.

Peer alcohol use was assessed as the mean of 4 items created for this study $(\alpha=.86)$. Participants used 7-point scales ("None of them" - "All of them") to indicate the proportion of [their friends / other people their age] who regularly [drink alcohol / get drunk]. 
Respondents used 7-point scales ("not at all" - "extremely") to indicate their belief in the effectiveness of eight alcohol control strategies chosen on the basis of variations in evidence of their effectiveness (Anderson, Chisholm \& Fuhr, 2009) and differences in their focus on individuals or the environment: Enforce the law against serving drunk people; Strictly monitor late-night licensed premises; Teach people skills for resisting pressure to drink; Restrict late-night alcohol sales; Increase the price of alcohol; Reduce the number of outlets that sell alcohol; Ban alcohol sponsorship of sporting events; Raise the legal drinking age.

The sample reflected the population in terms of ethnicity (Brighton \& Hove Local Information Service, 2011) and alcohol use (Office for National Statistics [ONS], 2012), but the sampling strategy meant that it over-represented students (ONS, 2013).

\section{$\underline{\text { Results }}$}

Multivariate multinomial logistic regression analysis indicated that membership of the four alcohol use groups was predicted by DRSE $(\mathrm{p}<.01)$, concern about health effects of alcohol use $(\mathrm{p}<.01)$, and perceived peer alcohol use $(\mathrm{p}<.01)$. This indicated that moderate drinking may be encouraged by: 1) raising concern about the health effects of alcohol; 2) challenging misperceived peer norms for heavy drinking; and 3) developing skills to resist personal or peer-influenced impulses to drink (Author, 2014a). Furthermore, when participants were asked which alcohol control strategies they believed would be effective, there was support for teaching people alcohol refusal skills: only enforcing the law against serving drunk people received significantly greater support (Author, 2014b). These findings suggest a need to identify, understand, and enhance alcohol refusal skills and strategies.

\section{Phase 2}

In Phase 2, non-drinkers and moderate-drinkers were treated as a valuable source of information about how to successfully manage situations that provide opportunities to drink excessively. Experiential qualitative methods were applied to: (1) understand how young people experience non- and moderate-drinking; and (2) identify choices and practices that participants had found to be successful. Data collection and analysis were designed to inform Phase 3 by creating a psychosocial framework for the experience of moderate drinking and by generating evidence-based content for the intervention materials.

\section{Methods}

Semi-structured interviews lasting 40 to 110 minutes were conducted with 25 young people purposively sampled from Phase 1 participants. The sample was designed to include sufficient numbers of: men and women: non-drinkers and moderate drinkers; and respondents above or below the legal alcohol purchase age of 18 years. People older than the target age 
group for the intervention (i.e., those aged 18-21) were included to allow analysis of: illicit and legal drinking; different drinking contexts; and how participants' understanding of their alcohol use changed over time. This allowed examination of the key issues as reported by the target group, and in the reflections and reminiscences of slightly older people. Interviews were grounded in participants' experiences of alcohol use and non-use. They explored motivations and influences related to (not) drinking; impact of (not) drinking on identity and social relationships; and use of specific strategies for non-drinking and moderate-drinking. Interviews were conducted by the second author (a 28 year old white woman) at the host university or the participant's host institution, transcribed verbatim, and anonymized.

Interpretative Phenomenological Analysis (Smith, Flowers \& Larkin, 2009) was conducted because it focuses on subjective experience and meaning-making and was therefore ideal for identifying advice on how to resist temptation/pressure to drink. Analysis focused on participants' experiences of implicit and explicit strategies concerning their own alcohol consumption, including thoughts, feelings, and behavior. It progressed iteratively through commonly accepted stages in IPA (Smith et al., 2009). Initial coding by the second author identified 104 codes that reflected distinct phenomena or experiences. These provided the basis of the interpretative phase of analysis: they were aggregated into six conceptually coherent themes which themselves clustered around a superordinate theme of the "sweet spot".

In IPA, assessment of reliability is not prescriptively quantified but instead reflects an iterative process in which all interpretation is clearly grounded in the raw data through procedures such as checking earlier transcripts against later analysis to consistent coding of themes. Yardley's (2000) four criteria for validity and quality were operationalized in a manner suggested by Smith et al. (2009). Sensitivity to context was demonstrated in our conceptualization of young people's alcohol use as a multiply-determined psychosocial phenomenon, and by our reflexivity in relation to data collection and analysis. Given that we were situated within the same broad cultural context as the participants, our interpretations will have been influenced by our own subjectivities. Throughout, we reflexively engaged in iterative processes of "setting aside" and "engaging with" our subjectivities by reflecting on our own experiences and interpretations and how these influenced the analytic process (Shaw, 2010). Commitment and rigor were promoted by having a selection of transcripts independently coded by the first author and second author to ensure that the interpretative process and outcomes were consistent. Transparency and coherence were demonstrated by explaining how themes were generated, presenting them in a clear way, and providing 
evidence to sow how these themes were grounded in the data. Impact and importance were demonstrated by showing how the analysis fed into intervention development in Phase 3. $\underline{\text { Results }}$

A detailed presentation of these findings is beyond the scope of this paper, but will be available elsewhere. Below we illustrate some key findings, and focus on the broad strategies and specific techniques identified by respondents. Analysis identified six major themes clustered around a central concept of the "Sweet Spot" of moderate drinking, exemplified by the following quote:

It's important to ... find that kind of sweet spot, between too sober and too drunk ... getting too drunk always tends to end kind of badly, but I ... like to get drunk enough open and friendly, but, kind of, sensible enough to not do anything too stupid ... there's something like satisfying about knowing that you're not drunk enough to do something, but, you're still, having a good time. [John, 18+, moderate]

John described the importance of knowing how alcohol affects him, and of knowing how to drink in ways that allow him to maximize the positive aspects of alcohol consumption and minimize the detrimental effects. His use of the phrase "sweet spot" neatly summarized both an experience and a desired (and desirable) state. The "Sweet Spot" (Figure 2) was defined as an experience or frame of mind in which an individual: feels good physically; feels in control; feels an authentic sense of belonging; feels satisfied with his/her choices to drink or not to drink; enjoys him/herself in drinking situations; and feels like him/herself when drinking. These themes related both to immediate experiences during drinking situations and to longer-term alcohol use. The central concept and its underpinning thematic dimensions represented broad strategies that could be enacted via specific tactics used to manage pressure/temptation to drink excessively.

$>$ Figure $2<$

For example, analysis identified the importance of "feeling like you can be who you are". Participants tended to value acting in ways that reflect who they are, regardless of the amount of alcohol consumed. However, they also sometimes experienced a disjunction between "real" choices made when sober and choices made "because I was drunk". This could be experienced positively as greater confidence or sociability: "you get sort of really happy. You're up for dancing and stuff' (Heather, 16-17, moderate). Nevertheless, many had also experienced a negative, jarring disjunction between behavior when drinking and authentic beliefs. Like other interviewees, Iris $(18+$ moderate $)$ noted that this disjunction prompted questions about the way she drank the way she did, especially when it led to interpersonal conflict. She experienced a tension between her feelings about whether she needs alcohol, 
and the effects of alcohol on what she values for herself and her relationships with others. This resulted in her deciding to reduce her alcohol intake:

I thought "Really? Do I want this [argument] to be happening?", and then to be feeling really low ... it's not that you become dependent, but sometimes - especially I think if you're a shy person or you lack a bit of self-confidence being with people - it's just like there easy thing to do ...[but] something which is meant to be fun, it's not any more.

These findings could be translated to interventions as the theme "feeling like you can be who you are", to reflect the broad strategy whereby individuals ensure that the choices they make while drinking are the ones they would want to make when sober. This suggests a need to support young people to be self-reflective using non-judgmental techniques. Specific tactics to allow this that were evident in the data included: "ask yourself if you want to meet the drunk version of yourself'.

\section{Phase 3}

Phase 3 entailed developing a video-based resource to promote responsible drinking among young people. The 11-minute pilot video "Hitting the Sweet Spot" was designed to illustrate successful alcohol management strategies (www.youtube.com/watch?v=7iGECfYahow). The target audience of 15-17 year old students and teachers of alcohol education was chosen because school-based education would provide contact with a broad range of young people at a time when many begin to use alcohol (ONS, 2012). The video was not designed to change behavior directly. It was anticipated that the video and associated lesson plans would be used within alcohol education to prompt individuals and groups to think critically about personal and social expectations and practices related to alcohol use.

\section{Resource Development}

The content and structure of the video were designed in line with best practice in health psychology and behavior change theory, and recent qualitative research into psychosocial aspects of alcohol use (Brown \& Gregg, 2012; de Visser et al., 2013; Jayne et al., 2010; Livingstone et al., 2011; MacNeela \& Bredin, 2011; Szmigin et al., 2011). The approach involved several different techniques identified in Abraham and Michie's (2008) taxonomy of behavior change intervention techniques - providing information about others' approval; prompting identification of barriers; providing general encouragement; modeling behavior; and providing opportunities for social comparison; providing information about consequences; teaching use of prompts/cues; and planning social support. It also employed the two aspects of peer education noted earlier: using peers as models and opinions leaders (Kelly et al, 1992); and encouraging young people to think critically about how their own behavior is influenced by a "binge drinking" youth culture (Campbell \& MacPhaill, 2002). 
The focus of the video was directed by the phenomenological themes identified in Phase 2, with each theme represented by a video segment (e.g., "Feeling like you can be who you are"). The strategies and tactics presented in the video were adapted from Phase 2 study findings (e.g., by creating an composite account of a non-drinker's experience of going to university). Phase 2 participants were invited to become actors in the video, and were invited to provide feedback on the video messages and production process.

Qualitative research has indicated that: long-term health effects are not sufficiently motivating to young people; extreme/shocking outcomes are dismissed as unlikely; a preaching/patronizing tone is counter-productive; and campaigns are often seen to be too obviously artificial/staged (de Visser et al., 2013; Fry, 2011; Harrison et al., 2011). Therefore, the video: used an informal "behind-the-scenes" style and a lighthearted tone to reduce defensiveness; presented non-drinkers and moderate drinkers as socially competent; and modeled effective strategies. An introductory voice-over made it clear that the "actors" in the video were young people who had participated in Phase 2 or other volunteers to help viewers to perceive the strategies as credible and feasible (Stephenson et al., 2004). The sixth author guided development of the video in light of his substantial experience of producing videos for late adolescents and young adult audiences.

\section{Resource Description}

The video was structured with a brief introduction, segments corresponding to three of the six identified themes in Figure 2 (feeling that you can be who you are; feeling good in the body; feeling safe and secure), and a conclusion reiterating the "Sweet Spot" message. Each segment used a combination of monologues, conversations and text, and employed both humorous and more serious tones to discuss a range of topics. The video included brief use of key statistics to highlight the prevalence of uncontrolled drinking (e.g., " 2 in 5 young people who got drunk in the last month didn't mean to"), rather than emphasizing health concerns or extreme/unlikely outcomes. Production of the video was accompanied by development of ideas for how to incorporate the videos in lesson plans for in-class use with secondary school students. This was carried out in consultation with school-based health education experts with the aim of evaluating these suggestions in Phase 4.

\section{Phase 4}

Phase 4 was a qualitative evaluation of the video developed in Phase 3. Whereas Phase 2 had an experiential focus and used individual interviews to generate suitable data, in this phase focus group discussions were used to generate: opinions of the video; suggestions for how to improve it; and to generate suggestions from students and teachers as to how a revised 
version of the video might be deployed in health education in line with concerns about alcohol education highlighted in past research (de Visser et al., 2013; Jayne et al., 2010; Szmigin et al., 2011) and in Phase 2. Focus groups were considered the appropriate methodology because they allow examination of interaction between research participants as well as similarities and differences in opinions, and because of the project's focus on drinking as a social phenomenon (Kitzinger, 1994). Focus groups allowed examination of consensus and disagreement about the overall concept and specific elements. Analysis of participants' reception of the "sweet spot" concept also served as an indicator of the validity and transferability of the Phase 2 findings (Yardley, 2000).

Methods

Focus groups were conducted to elicit opinions about the pilot video and lesson plans from key stakeholders. Data collection was carried out in five schools selected to include urban and semi-rural schools, government and independent schools, and a Pupil Referral Unit (PRU: a government educational institution for children who are excluded, ill, or otherwise unable to attend other schools). Thirteen group discussions ranging in size from 3-9 participants were conducted with students aged 15-17. Five focus groups ranging in size from 2-5 participants were conducted with teachers: these included Personal, Social\& Health Education (PSHE) specialists, teachers of science and health promotion, as well as head teachers and deputy heads. The discussions were facilitated by a 28 year old white women and/or a 40 year old white man.

Participants were shown the video in a classroom setting, and invited to give their opinions in several domains:

- Content: understanding of, and reactions to, the "sweet spot" message; perceived utility of the message and videos;

- Style: perceptions of the behind-the-scenes approach, visual style, and use of non-actors

- Implementation: comparison to existing alcohol education resources; beliefs about how the video could be used with other classroom activities.

- Improvements: including suggested revisions; and preferred mode of delivery Focus groups were audio-recorded and transcribed verbatim. They then underwent structured Thematic Analysis (Braun \& Clarke, 2006), a method chosen to enable identification of themes related to the four domains noted above, and suggestions for improving the video. The second author analyzed group discussions involving students. The first author analyzed group discussions involving PSHE teachers and verified the second author's coding of transcripts. An iterative analytic process identified codes on the basis of prevalence and 
relevance, and these were grouped into pre-identified themes according to the four domains identified above to ensure a balance between pre-identified topics and emergent issues (Braun \& Clarke, 2006; Fereday \& Muir-Cochrane, 2006).

As in Phase 2, Yardley's (2000) validity and quality criteria were applied. Sensitivity to context was demonstrated in our awareness of young people's alcohol use as a legal, social, and health issue, and by our reflexivity during data collection and analysis. Because we shared the same broad cultural context as the teachers and students, we reflexively engaged in processes of "setting aside" and "engaging with" our subjectivities and reflecting on how our own beliefs and experiences may have influenced the analytic process (Shaw, 2010). We also critically reflected on how student participants sometimes enacted social pressures and expectations addressed by the video. Commitment and rigor were promoted by the first and second authors conferring after conducting initial independent coding to ensure a consistent approach to analysis and interpretation. Transparency and coherence were demonstrated by explaining how themes were generated, presenting them in a clear way, and providing evidence to show how these themes were grounded in the data. Impact and importance were apparent in students' and teachers' responses to the video and their suggestions for how to improve it.

\section{Results}

The data provided valuable information about responses to the video and identified several aspects to address to ensure that it would be acceptable and useful in schools.

Content. The content was understood by the student participants, who considered the "sweet spot" a realistic, useful behavioral framework: they consistently reflected the message that "you don't need to drink that much to have a good time" (F student, school 1). As intended, they used social and personal frames for determining the sweet spot, as opposed to physical health messages. The video was seen to address DRSE and provide meaningful support for making healthy choices:

I think it was just sort of more like: "Think about it yourself and make your own decisions about how you want to drink and what you want to drink", but sort of advising about how to (M student, school 2)

Young people generally believed that the video realistically addressed important social contexts of drinking, contributors to drinking practices, and consequences of excess. They valued this approach as realistic because it incorporates real experiences, and acknowledges perceived benefits of drinking.

Young people noted that the ease of implementing the strategies in the video would 
depend upon the nature of drinking spaces, prior experience, and friendship groups. Some felt that younger adolescents needed personal experience of testing or exceeding alcohol intake limits in order to "then know when your balance is" (F teacher, school 3), and felt this would diminish message utility, whereas others found the "sweet spot" a positive message for young people to aspire to that should be introduced before drinking habits became established.

Several teachers questioned whether all young people would have the maturity to apply the principles and suggested a need for more "how to" advice, suggesting that they needed "more tips on how to not feel pressured by your peers" (F teacher, PRU).

Focus groups with teachers suggested that the video could be a valuable addition to alcohol education to empower students to enact healthy choices about alcohol use:

It was such a brilliant thing to hear, you know. That's a really positive look - Wow! You got something back by not [drinking excessively] ( $F$ teacher, school 3)

Style. Students generally felt that the design, narrative and structural elements of the video enhanced its believability, realism and usefulness. They compared it favorably to existing resources believed to feature uncontextualized facts, messages of abstinence, and situations that are "almost so extreme that you just thought: 'that's not going to happen."' (M student, school 3). The video was perceived to engage with young people in a way that "doesn't like make you rebel - in a way it just makes you, like, understand it more" (F student, school 3) through its use of other young people, upbeat tone, and emphasis on drinking in moderation:

There's a kind of really good ... social aspect to it, so not so much about the facts and statistics but the fact that they said friends - so you can go out with friends and have a good time without getting drunk, or you can go out with friends and make sure that you have friends with you - so have a really good social aspect to it (F student, school 2)

Teachers also liked the "friendly", informal, behind-the-scenes style:

The fact that you used real people is brilliant (F teacher, PRU)

However, some teachers and students felt that the people in the video sometimes seemed "awkward", and the interactions "staged". They acknowledged, however, that it is difficult to get past the "contrived" nature of video production.

Implementation. Students felt that video addressed young people's lived experiences in a more compelling and useful way than existing approaches, and addressed a need for resources that could help them to make healthy choices. Students and teachers consistently suggested that the video would be best used to prompt and/or focus discussions of opinions, strategies and capacities in small-group collaborative learning contexts:

I think like a discussion, talk about the best way to like go to a party without getting drunk. Like, here are some examples, and then show them the video. (M student, school 2) 
Students' and teachers' preference for using the video to prompt reflection and prepare for social situations matched a psychosocial resilience-based approach: rather than simply being told something, students would be actively encouraged to develop their own strategies. Although teachers understood and appreciated the "sweet spot" message, several noted that the harm reduction message may be "quite hard" to use because it could be seen to entail encouragement of illegal activity:

I wouldn't want you telling my Year 9 or Year 10 [13-15 year old] actually to go and drink to their sweet spot ( $F$ teacher, school 1)

Teachers also noted that video-based discussion may lead to students disclosing information about illegal activity, and that teachers would have to "be careful" with this. Although there was no firm consensus on an appropriate target age, participants suggested that the video was best aimed at 15-17 year olds as part of efforts to prevent excessive consumption among people who are exploring drinking but have not yet established routines.

Students and teachers felt that in addition to having copies of materials for in-class use, it would be useful to have a website This could facilitate in school use across multiple classes to stimulate group work, and would also enable individual use outside of class time.

Improvements. Some teachers suggested that more attention could be given to describing the negative consequences of heavy drinking, not just alluding to these. It was suggested that stories about bad experiences would help viewers relate to them because "we all love a story" (female teacher, school 1) and such stories would boost the perceived "honesty" of the videos. Similarly, some students wanted to hear more personal accounts of the negative impact of excessive drinking upon characters' personal and social lives. Many students also expressed a desire for more details of how the people in the video lived out the healthy choices they talked about on screen. One option suggested by students would be to provide brief background stories for each person on the website.

Like if someone, one of the people in that told, like, a really, like, story about exactly what happened to them and stuff, then it'd probably, like, draw you in. You'd probably go away and, like, remember it, nod, and think, "Oh, that's really bad" (F student, College 2)

Students and teachers suggested that in order to effectively target under-18 year olds, future resources should include discussion of experiences in informal drinking spaces such as house parties, parks and streets rather than bars. They also suggested that the video should include a broader range of people than the perceived "educated", "middle-class" young people in the video. Students and teachers felt that the segments involving two or more people could be made to seem less awkward by including real friendship groups talking about 
positive shared experiences of non-drinking or moderate alcohol use.

It was clear from these findings that a revised version of the video and associated lesson plans would be a valuable addition to school-based alcohol education materials.

\section{Discussion}

This paper has illustrated how different qualitative methods may be used as part of a coordinated systematic approach the development and refinement of health psychology interventions (Kotler \& Lee, 2011). Qualitative methods contributed to the development of the videos in three key ways. First, the results of recent qualitative research highlighted a need for interventions that better address the concerns and motivations of young people and that use messages and media that are appealing to them (Brown \& Gregg, 2012; de Visser et al., 2013; Fry, 2011; Harrison et al., 2011; Hutton, 2012; Jayne et al., 2010; Livingstone, Young \& Manstead, 2011; Szmigin et al., 2011).

Second, use of experiential qualitative methods in Phase 2 facilitated identification of specific examples of how young people can put into practice generic advice to "drink responsibly", and thereby address an absence in current public health interventions. The concept of the "Sweet Spot" encapsulated an experiential understanding of young people's moderate- and non-drinking, and offered a substantive definition of how moderate drinking may be experienced and enacted. This concept and its related associated behavioral strategies: address young people's concerns and resilience capacities as they navigate social drinking situations; specifies targets for health promotion efforts; and generates realistic goals and guidance. An important aspect of the Sweet Spot is that it is subjectively defined and may be an important complement to tactics focusing on consumption (e.g., monitoring or counting "units" or "standard drinks") or avoidance of alcohol. The Phase 2 interviews provided rich examples of behavioral strategies and tactics used successfully by young people which were then used to inform development of the video in Phase 3.

The use of qualitative methods in Phase 4 allowed assessment of the likely classroom utility of the videos developed in Phase 3, and provided valuable suggestions for how the materials could be improved to make them more acceptable to, and useful for, young people and teachers. Students and teachers felt that the video could make a valuable addition to existing alcohol education resources, and that lessons involving group work could be planned around the video content. An important aspect of this evaluation was to determine the likely acceptability of the intervention to schools given that the intervention was based on a harm reduction philosophy that does not necessarily advocate abstinence (Midford et al., 2012; Lenton \& Midford, 1996). Notably, some teachers questioned whether all parents and schools 
would feel comfortable endorsing the "Sweet Spot" message: some may consider it to be an invitation to inexperienced young people to drink in potentially harmful ways (although the "Sweet Spot" message does encourage personal and group safety). The apparent ambivalence in the message reflects ambivalence toward alcohol-related harm observed at the individual level (e.g., de Visser \& Smith, 2007a), and at a broader social level (Babor et al., 2010). However, it is important to note that many parents may respond positively to approaches to alcohol education that are not solely focused on abstinence (Gilligan \& Kypri, 2012). The acceptance of harm reduction approaches may reflect awareness that underage drinking is not simply a legal issue, but rather an issue that has implications for young people's physical and psychosocial well-being.

It is not anticipated that the "Sweet Spot" intervention will be appropriate for all contexts, or that it will be a panacea for problematic alcohol use among young people. It is therefore important to consider where it might fit within broader alcohol control strategies (Anderson et al., 2009). Interventions that target individual behaviour - so-called "downstream" approaches - tend to have less impact on risk behavior than do "upstream" approaches that focus on policy and regulation (Anderson et al., 2009; Hoek \& Jones, 2011). Although the intervention described and evaluated here focused on the social context and individual capacities - and could be considered more "upstream" than other "downstream" approaches (Hoek \& Jones, 2011) - it would still need to incorporated with more "upstream approaches. Here it is worth noting that the Phase 1 data revealed broad support among young people for a range of population-level policies as well as individual-level strategies (Author, 2014b).

To summarize the mixed method approach applied here, the quantitative data generated in Phase 1 confirmed the potential impact of an intervention designed to enhance DRSE, informed the focus of the interviews conducted in Phase 2, and provided the purposive sample of interviewees for these. The qualitative data generated in Phase 2 revealed the importance of the "sweet spot" concept and provided experience-based content for the "sweet spot" video developed in Phase 3. The qualitative data generated in Phase 4 confirmed the potential utility of the "sweet spot" video resource, and provided ideas for how to improve the resource and how best to incorporate it in alcohol education. Using the findings of Phase 2 to develop the intervention ensured that strategies were grounded in the lived experience of the target audience. By treating participants as "experts", experiential methods allowed the identification of strategies, tactics and tensions unanticipated by other approaches. Young people were also treated as experts by including them in the presentation of the intervention. As suggested in Phase 4, this maximized the applicability of the intervention to a target 
audience of peers (Kelly et al., 1992; Starkey et al., 2009). There is a confluence between resilience-based approaches that seek to promote change by nurturing young people's strengths, and experiential qualitative methods that provide analytical space for identifying, theorizing and exploiting those strengths.

The four phases described here reflected social marketing approaches (Kotler \& Lee, 2011) and were designed to lead to future work to revise and finalize the video, and to develop lesson plans and materials needed for best use of the video in classrooms (Figure 1). The video was developed to include a number of the techniques identified in Abraham and Michie's (2008) taxonomy of behavior change intervention techniques, including: providing information about others' approval; prompting identification of barriers; modeling behavior; and planning social support. It also employed two important aspects of peer education: using peers as opinions leaders and models in relation to moderate alcohol use (Kelly et al, 1992); and encouraging young people to think critically about their own behavior in a "binge drinking” youth culture (Campbell \& MacPhaill, 2002).

Although this paper has argued for research programs that combine methods, it must be noted that mixed-method research can produce epistemological tensions between methods based on different philosophical foundations (Denzin \& Lincoln, 2005). However, applied health researchers may prioritize a pragmatic approach to finding out what works, and treat research methods more as tools or techniques than strict epistemological frameworks (Denzin \& Lincoln, 2005; Tashakkori \& Teddlie, 2003). A pragmatic approach suggests that methods should be combined in ways that exploit their strengths and acknowledge their limitations (Greene \& Caracelli, 1997). This requires expertise across methods to prevent inappropriate extension of findings or linkages between phases, but yields the benefits of a multidimensional research program targeting different aspects of intervention development.

Funding has been secured to conduct the first phases of the "future work" identified in Figure 1. Such work is required to evaluate the efficacy of the intervention (as opposed to the earlier stages of evaluating whether the intervention concept was feasible). First, the video is being revised based on Phase 4 findings. Existing scenes have been revised or deleted, and new scenes are being added so that the video addresses all six thematic components of the "Sweet Spot". Lesson plans, taking into account Phase 4 findings are being developed in collaboration with a health education expert. A website will be developed to host the videos and lesson plans for use within and outside of class time. Second, a feasibility study will be conducted to verify methods of data collection, confirm expected response rates, and identify any issues related to intervention delivery that require modification before a full trial. It is 
important to note that the data collection and analysis - and therefore the materials that were developed - were context-specific. The findings reported here and the intervention materials that were developed may not necessarily be applicable in other contexts without thorough testing of the appropriateness of the message and the medium (Kotler \& Lee, 2011).

There is a need for interventions that help young people to put into practice advice to choose healthy behaviors. The approach to developing, refining, and evaluating health promotion materials described in this paper - i.e., based on identifying which behavioral strategies and interventions young people find to be acceptable and effective - could be applied to other social behaviors that have important health consequences, such as smoking, and sexual risk behavior. The material presented here highlights the integral role of qualitative methods in coordinated structured approaches to developing and refining interventions that can make a difference to young people's health behavior.

\section{References}

Abraham, C. \& Michie S. (2008). A taxonomy of behavior change techniques used in interventions. Health Psychology, 27, 379-387. doi:10.1037/0278-6133.27.3.379

Anderson, P., Chisholm, D., Fuhr, D.C. (2009). Effectiveness and cost-effectiveness of policies and programmes to reduce the harm caused by alcohol. Lancet, 373, 2234-2246. doi: 10.1016/S01406736(09)60744-3.

Babor, T.F., Caetano, R., Casswell, S, Edwards, G., Giesbrecht, N., ... Rossow, I. (2010) Alcohol: No Ordinary Commodity Research and Public Policy (2nd ed). Oxford: Oxford University Press.

Babor, T.F., Higgins-Biddle, J.C., Saunders, J.B., \& Monteiro, M.G. (2001). The Alcohol Use Disorders Identification Test: Guidelines for Use in Primary Care $\left(2^{\text {nd }}\right.$ Edition). Geneva: World Health Organization.

Boles, S.M., \& Miotto, K. (2003). Substance abuse and violence: A review of the literature. Aggression \& Violent Behavior, 8, 155-174. doi:10.1016/S1359-1789(01)00057-X

Bonell, C., Parry, W., Wells, H., Jamal, F., Fletcher, A., Harden, A., Thomas, J., Campbell, R., Petticrew, M., Murphy, S., Whitehead, M. \& Moore, L. (2013). The effects of the school environment on student health: A systematic review of multi-level studies. Health \& Place, 21, 180-191. doi: 10.1016/j.healthplace.2012.12.001

Borsari, B. \& Carey, K.B. (2003). Descriptive and injunctive norms in college drinking: a metaanalytic integration. Journal of Studies on Alcohol, 64, 331-341.

Braun, V., \& Clarke, V. (2006). Using thematic analysis in psychology. Qualitative Research in Psychology, 3, 77-101.

Brighton \& Hove Local Information Service (2011). Profile library. Online resource available at http://www.bhlis.org/profiles/ (last visited on 12/02/2013).

Brown, R., \& Gregg, M. (2012). The Pedagogy of Regret: Facebook, binge drinking and young women. Continuum, 26,357-369. doi:10.1080/10304312.2012.665834

Campbell, C. \& MacPhail, C. (2002) Peer Education, Gender and the Development of Critical Consciousness. Social Science \& Medicine, 55, 331-345.

Cheng, H. Kotler, P. \& Lee, N.R. (2011). Social Marketing for Public Health: Global Trends and Success Stories. Sudbury, MA: Jones \& Barlett.

Cherpitel, C.J., Bond, J., Ye, Y., Borges, G., MacDonald, S., \& Giesbrecht, N. (2003). A crossnational meta-analysis of alcohol and injury. Addiction, 98, 1277-1286. doi:10.1046/j.1360- 
0443.2003.00459.x

Conroy, D. \& de Visser, R.O. (in press). 'Man up!': Discursive constructions of non-drinkers among UK undergraduates. Journal of Health Psychology. doi:10.1177/1359105312463586

de Visser, R.O. \& McDonnell, E.J. (2012). “That's OK. He's a guy": A mixed-methods study of gender double-standards for alcohol use. Psychology \& Health, 27, 618-639.

doi:10.1080/08870446.2011.617444

de Visser, R.O. \& Smith, J.A. (2006). Mister in between: a case study of masculine identity and health-related behaviour. Journal of Health Psychology, 11, 685-695. doi: $10.1177 / 1359105306066624$

de Visser, R.O. \& Smith, J.A. (2007a). Young men men's ambivalence toward alcohol. Social Science \& Medicine, 64, 350-362. doi:10.1016/j.socscimed.2006.09.010

de Visser, R.O. \& Smith, J.A. (2007b). Alcohol consumption and masculine identity among young men. Psychology \& Health, 22, 595-614. doi:10.1080/14768320600941772

de Visser, R.O., Smith, A.M.A. \& Richters, J. (2005). Can we generalise to the broader population from studies of sexual behaviour among university students? Australian \& New Zealand Journal of Public Health, 29, 436-441. doi:10.1111/j.1467-842X.2005.tb00223.x

de Visser, R.O., Wheeler, Z., Abraham, S.C.S. \& Smith, J.A. (2013). 'Drinking is our modern way of bonding': young people's beliefs about interventions to encourage moderate drinking. Psychology \& Health, 28, 1460-1480. doi: 10.1080/08870446.2013.828293.

Denzin, N.K., \& Lincoln, Y.S. (Eds.) (2005). Handbook of Qualitative Research (3rd ed). London: Sage.

Fereday, J. \& Muir-Cochrane, E. (2006). Demonstrating rigor using thematic analysis: a hybrid approach of inductive and deductive coding and theme development. International Journal of Qualitative Methods, 5, 1-11.

Fisher, J.D., Fisher, W.A., Williams, S.S. \& Malloy, T.E. (1994). Empirical tests of an informationmotivation-behavioral skills model of AIDS-preventive behavior with gay men and heterosexual university students. Health Psychology, 13, 238-250. doi: 10.1037/0278-6133.13.3.238

França, L.R., Dautzenberg, B. \& Reynaud, M. (2010). Heavy episodic drinking and alcohol consumption in French colleges: the role of perceived social norms. Alcohol: Clinical \& Experimental Research, 34, 164-174.

Fry, M.L. (2010). "Seeking the pleasure zone": Understanding young adult's intoxication culture. Australasian Marketing Journal, 19, 65-70. doi:10.1016/j.ausmj.2010.11.009

Gilligan, C. \& Kypri, K. (2012) Parent attitudes, family dynamics and adolescent drinking: qualitative study of the Australian parenting guidelines for adolescent alcohol use. BMC Public Health 2012 12:491. doi:10.1186/1471-2458-12-491

Gordon, R., McDermott, L., Stead, M. \& Angus, K. (2006). The effectiveness of social marketing interventions for health improvement: What's the evidence? Public Health, 120, 1133-1139. doi:10.1016/j.puhe.2006.10.008

Greene, J.C., \& Caracelli, V.J. (1997). Defining and describing the paradigm issue in mixedmethod evaluation. New Directions for Evaluation, 74, 5-17. doi:10.1002/ev.1068

Griffin, C., Bengry-Howell, A., Hackley, C., Mistral, W., \& Szmigin, I. (2009). 'Every time I do it I absolutely annihilate myself': Loss of (self-)consciousness and loss of memory in young people's drinking narratives. Sociology, 43, 457-476. doi:10.1177/0038038509103201

Harrison, L., Kelly, P., Lindsay, J., Advocat, J., \& Hickey, C. (2011). "I don't know anyone that has two drinks a day": Young people, alcohol and the government of pleasure. Health, Risk \& Society, 13, 469-486. doi:10.1080/13698575.2011.596190

Hendershot, C.S., Witkiewitz, K., George, W.H. \& Marlatt, G.A. (2011). Relapse prevention for addictive behaviors. Substance Abuse Treatment, Prevention, and Policy, 6: 17. doi:10.1186/1747597X-6-17 
Herring, R., Berridge, V. \& Thom, B. (2008). Binge drinking: An exploration of a confused concept. Journal of Epidemiology and Community Health, 62, 476-479.

doi:10.1136/jech.2006.056721

Hoek, J. \& Jones, S. (2011). Regulation, public health and social marketing: a behaviour change trinity. Journal of Social Marketing, 1, 32-44. doi: 10.1108/20426761111104419

Hutton, F. (2012). Harm reduction, students and pleasure: An examination of student responses to a binge drinking campaign. International Journal of Drug Policy, 23, 229-235. doi:10.1016/j.drugpo.2011.10.001

Jayne, M., Valentine, G. \& Holloway, S.L. (2010) Emotional, embodied and affective geographies of alcohol, drinking and drunkenness. Transactions of the Institute of British Geographers, 35, 540554. doi:10.1111/j.1475-5661.2010.00401.x

Kelly, J. A., St Lawrence, J. S., Stevenson, L. Y., Hauth, A. C., Kalichman, S. C., Diaz, Y. E., Brasfield, T. L., Koob, J. J., \& Morgan, M. G. (1992). Community AIDS/HIV risk reduction: the effects of endorsements by popular people in three cities. American Journal of Public Health, 82, 1483-1489.

Kitzinger, J. (1994). The methodology of focus groups: the importance of interaction between research participants. Sociology of Health \& Illness, 16, 103-121.

Larimer, M.E., Palmer, R.S., Marlatt, G.A. (1999) Relapse prevention. An overview of Marlatt's cognitive-behavioral model. Alcohol Research \& Health, 23, 151-160.

Lenton, S. \& Midford, R: (1996) Clarifying 'harm reduction'? Drug \& Alcohol Review, 15, 411413.

Lewin, S., Glenton, C., Oxman, A.D. (2009) Use of qualitative methods alongside randomised controlled trials of complex healthcare interventions: methodological study.

British Medical Journal, 339, b3496. doi: http://dx.doi.org/10.1136/bmj.b3496

Livingstone, A.G., Young, H. \& Manstead, A.S.R. (2011). "We drink, therefore we are": the role of group identification and norms in sustaining and challenging heavy drinking "culture". Group Processes \& Intergroup Relations, 14, 637-649. doi:10.1177/1368430210392399

MacNeela, P., \& Bredin, O. (2011). Keeping your balance: Freedom and regulation in female university students' drinking practices. Journal of Health Psychology, 16, 284-293. doi:10.1177/1359105310372977

MacPherson, I. \& McKie, L. (2010). Qualitative Research in Programme Evaluation. In: I. Bourgeault, R. Dingwall, R. \& R. de Vries (eds) Sage Handbook on Qualitative Methods in Health Research. London: Sage.

Midford, R., Cahill, H., Foxcroft, D., Lester, L., Venning, L., Ramsden, R. \& Pose, M. (2012) Drug education in Victorian schools (DEVS): the study protocol for a harm reduction focused school drug education trial. BMC Public Health, 12:112. [doi: doi:10.1186/1471-2458-12-112]

NHS Change 4 Life (2013). Choose Less Booze: Easy Drink Swaps. Retrieved 10 May 2013 from http://www.nhs.uk/change4life/pages/alcohol-drink-swaps.aspx

Oakley, A., Strange, V., Bonell, C., Allen, E., Stephenson, J. \& RIPPLE Study Team. (2006). Process evaluation in randomised controlled trials of complex interventions. British Medical Journal, 332, 413-416.

Office for National Statistics (2012). General Lifestyle Survey, 2011. Newport: Office for National Statistics.

Office for National Statistics (2013). Table A06: Educational status and labour market status for people aged from 16 to 24. Newport: Office for National Statistics, 2013. Available at: www.ons.gov.uk/ons/rel/lms/labour-market-statistics/december-2013/table-a06.xls

Randolph, W. \& Viswanath, K. (2004). Lessons learned from public health mass media campaigns: marketing health in a crowded media world. Annual Review of Public Health, 25, 419437. DOI: 10.1146/annurev.publhealth.25.101802.123046 
Regan, D. \& Morrison, T. (2013). Adolescents' negative attitudes towards non-drinkers: a novel predictor of risky drinking. Journal of Health Psychology, 18, 1465-1477. doi: $10.1177 / 1359105312464676$

Rutter, M. (1990). Psychosocial resilience and protective mechanisms. In J. Rolf, A.S. Masten, D. Cicchetti, K.H. Neuchterlein \& S. Weintraub (eds.) Risk and Protective Factors in the Development of Psychopathology (pp.181-212). Cambridge: Cambridge University Press.

Sargeant, J. (2012). Qualitative research part II: Participants, analysis, and quality assurance. Journal of Graduate Medical Education, 4, 1-3.

Shaw, R. (2010). Embedding reflexivity within experiential qualitative psychology. Qualitative Research in Psychology, 7, 233-243. doi: 10.1080/14780880802699092

Smith, J.A., Flowers, P. \& Larkin, M. (2009). Interpretative Phenomenological Analysis: Theory Method and Research. London: Sage.

Starkey, F., Audrey, S., Holliday, J., Moore, L. \& Campbell, R. (2009) Identifying influential young people to undertake effective peer-led health promotion: the example of A Stop Smoking In Schools Trial (ASSIST) Health Education Research, 24, 977-988.

Stephenson, J.M., Strange, V., Forrest, S., Oakley, A., Copas, A., ... and the RIPPLE study team (2004). Pupil-led sex education in England (RIPPLE study). Lancet, 364, 338-346.

Szmigin, I., Bengry-Howell, A., Griffin, C., Hackley, C., \& Mistral, W. (2011). Social marketing, individual responsibility and the "culture of intoxication". European Journal of Marketing, 45, 759779. doi:10.1108/03090561111120028

Szmigin, I., Griffin, C., Mistral, W., Bengry-Howell, A., Weale, L., \& Hackley, C. (2008). Reframing 'binge drinking' as calculated hedonism: Empirical evidence from the UK. International Journal of Drug, 19, 359-366. doi:10.1016/j.drugpo.2007.08.009

Tashakkori, A., \& Teddlie, C.B. (2003). Handbook of Mixed Methods in Social and Behavioural Research. Thousand Oaks, CA: Sage.

Thurnell-Read, T. (2011). Off the leash and out of control: masculinities and embodiment in Eastern European stag tourism. Sociology, 45, 977-991. doi:10.1177/0038038511416149

Tuong, W., Larsen, E.R. \& Armstrong, A.W. (2014). Videos to influence: a systematic review of effectiveness of video-based education in modifying health behaviors. Journal of Behavioral Medicine, 37, 218-233. doi: 10.1007/s10865-012-9480-7

White, A., de Sousa, B., de Visser, R., Hogston, R., Madsen, S.A, ..., Zatonski, W. (2011). The State of Men's Health in Europe. Brussels: European Commission.

Yardley, L. (2000). Dilemmas in qualitative health research. Psychology \& Health, 15, 215-228. doi: 10.1080/08870440008400302 
Figure 1 Outline of phases of evidence-based intervention development work

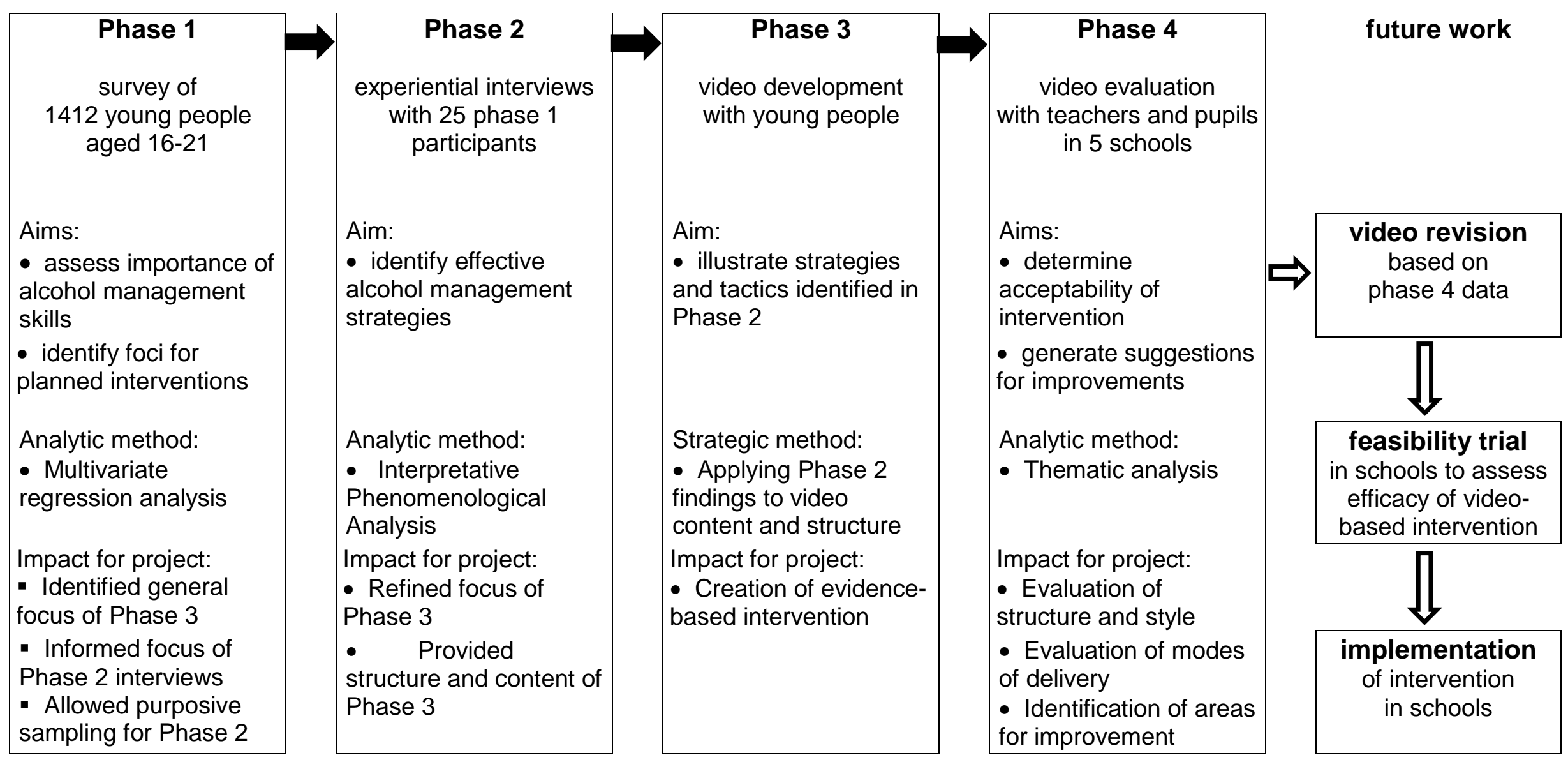


Theme description

enjoying the people

one is with, and

having a good time

in drinking

situations

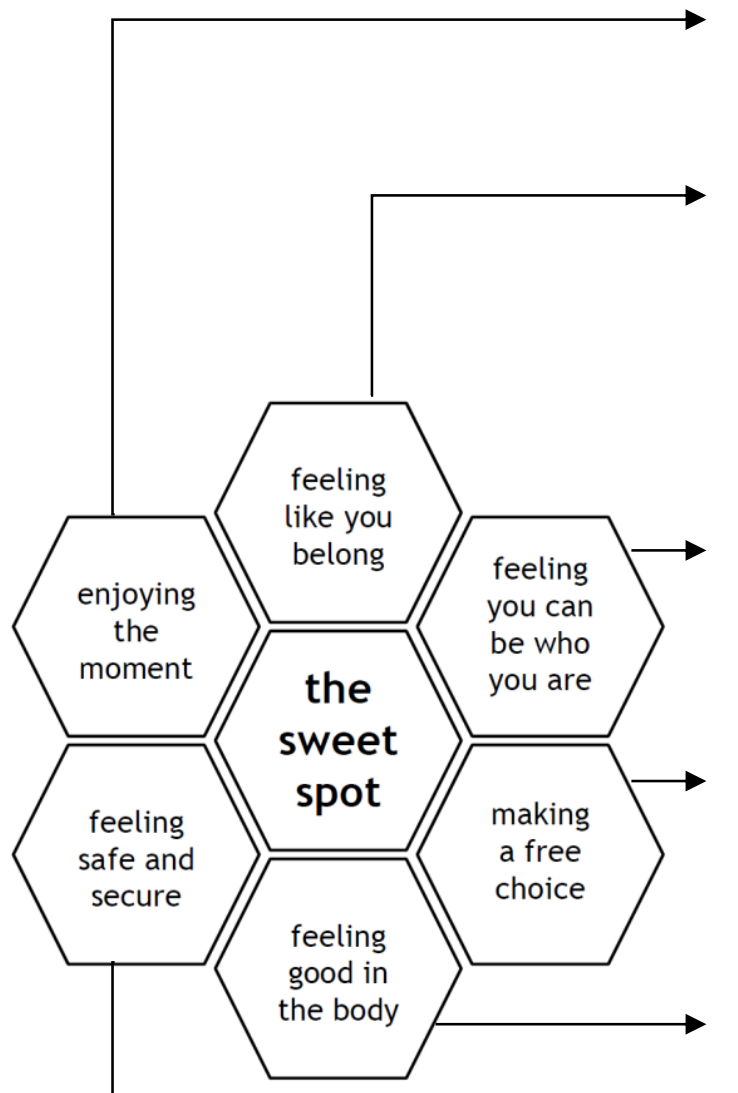

feeling authentically connected to, and

accepted by, one's

social network

feeling like oneself and behaving in ways that reflect

ne's identity and

values

feeling that the decisions one makes about drinking, and when drinking, are truly

one's own

maintaining feelings of physical wellbeing before,

during, and after drinking

a fundamental sense of control of oneself and the environment (including managed loss of control)

\section{Example quote}

"There's no need to get yourself into that state. It's not enjoyable. You're gonna get taken home, miss out" (F 16-17, moderate drinke

"It's not like our friendship was based on alcohol. We were friends before we started drinking" (M 18+, former drinker)

"My friends say they wouldn't want to meet the drunk version of themselves. I don't like the idea of there being a part of me that I wouldn't want to know fully

(F 18+, non-drinker)

"[We] literally have no idea what happened ... went out with the same intention to stay sober, and the opposite happened"

$$
\text { (F 16-17, moderate drinker) } \quad \text { choices you make }
$$

"The drinks are usually these kind of big, massive pints which re usually horrible ... I like to have a beer that tastes nice"

(M 18+, moderate drinker)

"If I get drunk [other people may] make me do something silly and embarrass me ... so I'm not going to drink"

\footnotetext{
(M 16-17, moderate drinker)
}

Characteristic strategies

Have a range of

engaging friends and interests - $\quad$ focus on other parts of a social event like music dancing, and talking to friends

Trust that your friends will generally accept and support your decisions

when drinking

kare of each other

\section{Example tactics}

Choose social activities that don't involve alcohol (e.g., meet friends in a cafe) unobtrusive non-alcoholic drinks (e.g., soft drinks) at social events

\section{Share your} trusted friend

- Reflect on whether the decisions you make when drinking are those you would make when sober

- Think about hidden rules and expectations about drinking in your social group choices you make

Drink only what tastes good, but drink less of it

- $\quad$ Slow down or stop drinking when you feel good fitness and mood

Check in on day-to-da friends' behavior when they you actually enjoy the taste of, but drink fewer of them

- Plan ahead, especially first drinking experiences - $\quad$ Plan ahead to avoid uncontrollable or frightening situations
Stay sober and

see what difference it makes

Observe your are drinking

\section{- $\quad$ Choose drinks}

\section{- Ask yourself} (

- Have an "exit strategy" to let you get home safely 